\title{
Review of the species of Stenothemus from Southeast China (Coleoptera, Cantharidae)
}

\author{
Yuxia YANG 1,*, Shujuan $\mathrm{GE}^{2}$, Xingke YANG ${ }^{3}$ \& Haoyu LIU ${ }^{4, *}$ \\ ${ }^{1,2,4}$ The Key Laboratory of Zoological Systematics and Application, School of Life Science, Institute \\ of Life Science and Green Development, Hebei University, Baoding 071002, Hebei Province, China. \\ ${ }^{3}$ Key Laboratory of Zoological Systematics and Evolution, Institute of Zoology, \\ Chinese Academy of Sciences, Beijing 100101, China. \\ *Corresponding authors: yxyang@hbu.edu.cn, liuhy@aliyun.com \\ 2Email:1264084679@qq.com \\ ${ }^{3}$ Email: yangxk@ioz.ac.cn \\ ${ }^{1}$ urn:lsid:zoobank.org:author:5DE2E8C5-0E72-4E81-979F-AA3596D4D9F5
${ }^{2}$ urn:lsid:zoobank.org:author:EDE4D3AD-FC52-4537-AFED-99272F0960BE
${ }^{3}$ urn:lsid:zoobank.org:author:8878D2C6-30E2-4C64-96EB-6BD8F03DB752
${ }^{4}$ urn:lsid:zoobank.org:author:9A257221-9F0A-4EC7-88E5-BC95AF249723
}

Abstract. The species of Stenothemus Bourgeois, 1907 from Southeast China are reviewed. Stenothemus fukienensis Wittmer, 1974 and S. kuatunensis Wittmer, 1979 are supplementarily described. Two new species are described, S. longicornis Y. Yang \& H. Liu sp. nov. (China: Guangdong) and S. flavus Y. Yang \& X. Yang sp. nov. (China: Zhejiang). Five new combinations are established: S. biimpressiceps (Pic, 1930) comb. nov. (from Cantharis L.), S. chinensis (Wittmer, 1982) comb. nov., S. limbatipennis (Pic, 1926) stat. rev. et comb. nov., S. nigriceps (Wittmer, 1955) comb. nov. and S. pallicolor (Wittmer, 1951) comb. nov. (from Lycocerus Gorham). Leiothorax atrosanguineus Švihla, 2005 syn. nov. is synonymized with $S$. chinensis, Lycocerus limatus Kazantsev, 2007 syn. nov. with S. limbatipennis. The above species are illustrated with habitus photos, aedeagi, abdominal sternites VIII and internal genitalia of female. A key for the identification of the above species is provided.

Keywords. Stenothemus, new species, new combination, new synonym, China.

Yang Y., Ge S., Yang X. \& Liu H. 2021. Review of the species of Stenothemus from Southeast China (Coleoptera, Cantharidae). European Journal of Taxonomy 744: 119-144. https://doi.org/10.5852/ejt.2021.744.1307

\section{Introduction}

The genus Stenothemus was proposed by Bourgeois (1907) based on Themus harmandi Bourgeois, 1902. It was revised by Wittmer (1974) and since then, the genus became better known. Later, some more species were added by Wittmer (1979), Okushima \& Satô (1997, 1999), Švihla (2004, 2005, 2011), Yang et al. (2014) and Hsiao et al. (2015, 2016). At present, it comprises 74 species in total, which are distributed in the Oriental and eastern Palaearctic regions. In China, 47 species are hitherto 
known. However, some species were originally described based on a single female or male type, which made them quite poorly known, and their status hard to clarify. During our study, the large series of specimens of Stenothemus at our disposal allowed us to review the genus based on the examination of the types. In the present study, the species from Southeast China are treated, and the others will be included in another paper.

\section{Material and methods}

The material studied is preserved in the following collections, and the primary types were returned to the collections from which they were borrowed or were otherwise deposited in public museums.

Genitalia of both sexes and abdominal sternites VIII of females were dissected and cleared in a olution of $10 \% \mathrm{NaOH}$, female genitalia were dyed with hematoxylin. Habitus photos were taken using a Leica M205A stereo microscope, multiple layers were stacked using Combine ZM (Helicon Focus 5.3). Line drawings were made using a camera lucida attached to a Nikon SMZ1500 stereo microscope, then edited in CorelDRAW 12 and Adobe Photoshop CS3.10.0.1.

Complete label data are listed for type specimens of the previously known species, using square brackets [ ] for our remarks and comments, [p] indicating that the following data are printed and [h] that they are handwritten. Quotation marks are used to separate data from different labels and a double slashes // to separate data from different lines of the same label. For additional specimens, quotation marks are used if their original labels are written in English.

Body length is measured from the anterior margin of the clypeus to the elytral apex and body width across the humeral part of elytra. Morphological terminology of female genitalia follows that of Brancucci (1980).
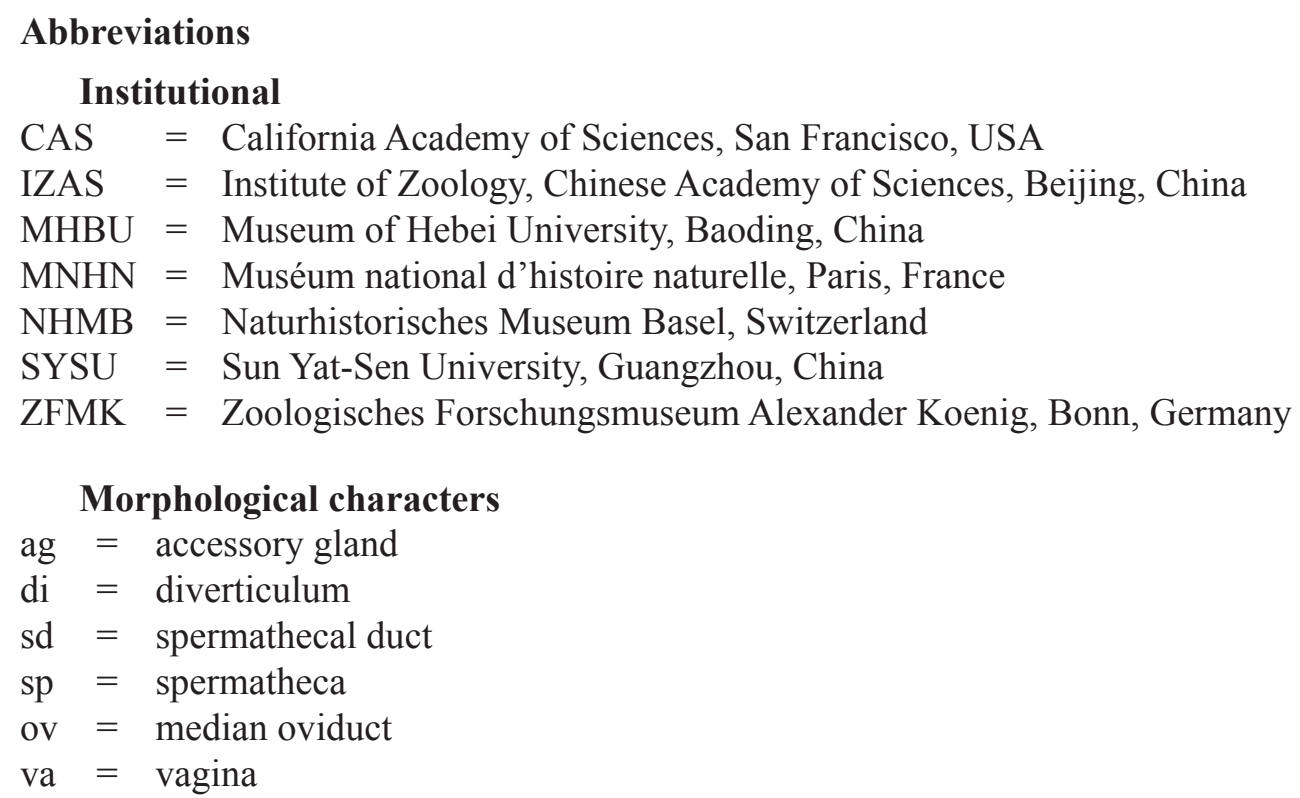


\section{Results}

\section{Taxonomic treatments}

Phylum Arthropoda Latreille, 1829

Class Insecta Linnaeus, 1758

Order Coleoptera Linnaeus, 1758

Superfamily Elateroidea Leach, 1815

Family Cantharidae Imhoff, 1856

Subfamily Cantharinae Imhoff, 1856

Genus Stenothemus Bourgeois, 1907

Stenothemus biimpressiceps (Pic, 1930) comb. nov.

Figs 1A, 2A, 3A-C, 6A, 8A

Cantharis biimpressiceps Pic, 1930: 78.

\section{Material examined}

\section{Holotype}

CHINA • O; [h] "Chine", [p] "81", [h-p] "Déterminé par//Heyden", [h] "sp.ignota", [h] "biimpressiceps//n. sp.", [p] "HOLOTYPUS", [h-p] "Stenothemus//biimpressiceps (Pic)// det. Y. X. Yang, 2009"; MNHN.

\section{Other material}

CHINA - Anhui • 1 ð̊; Yaoluoping National Natural Reserve; Jul. 2015; J. Fang leg.; MHBU. - Hubei

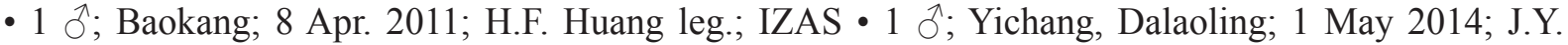
Su leg.; MHBU. - Jiangxi • 1 q; date and collector unknown; IZAS. - Zhejiang • 1 ภ, 2 q $\circ$; Anji, Longwangshan; $450 \mathrm{~m}$ a.s.1.; 11 May 1996; H. Wu leg.; IZAS • 1 ô; same locality and collector as for preceding; 12 May 1996; IZAS.

\section{Redescription}

MEAsurements. Body length (both sexes): 10.0-14.0 mm; width: $2.5-3.5 \mathrm{~mm}$.

Male (Fig. 2A)

Coloration. Body yellowish brown, sometimes with brown markings on disc of pronotum, mouthparts light brown, reddish brown at apices of mandibles, antennae, tarsi, tibiae and apices of femora dark brown. Body densely covered with yellow pubescence, sparsely on pronotum.

HEAD. Subquadrate, surface densely and finely punctate, each side with smooth and rectangular impression behind antennal socket; eyes strongly protruding, head width across eyes 1.2 times as wide as pronotum; terminal maxillary palpomeres long-triangular, widest in middle; antennae filiform, extending to twothirds length of elytra; antennomeres II about 1.5 times as long as wide at apices, III about 1.5 times as long as II, VII longest, XI slightly longer than X, pointed at apex.

Pronotum. Subrounded, nearly as wide as long, anterior margin arcuate, anterior angles rounded, lateral margins sinuate, posterior margin nearly straight and narrowly bordered, posterior angles sharp and protruding, disc distinctly convex on posterolateral parts, surface finely and densely punctate.

ELYTRA. Nearly parallel-sided, about 3.5 times as long as humeral width, 5.2 times as long as pronotum, surface finely and densely punctate, with weakly indicated longitudinal costae. 

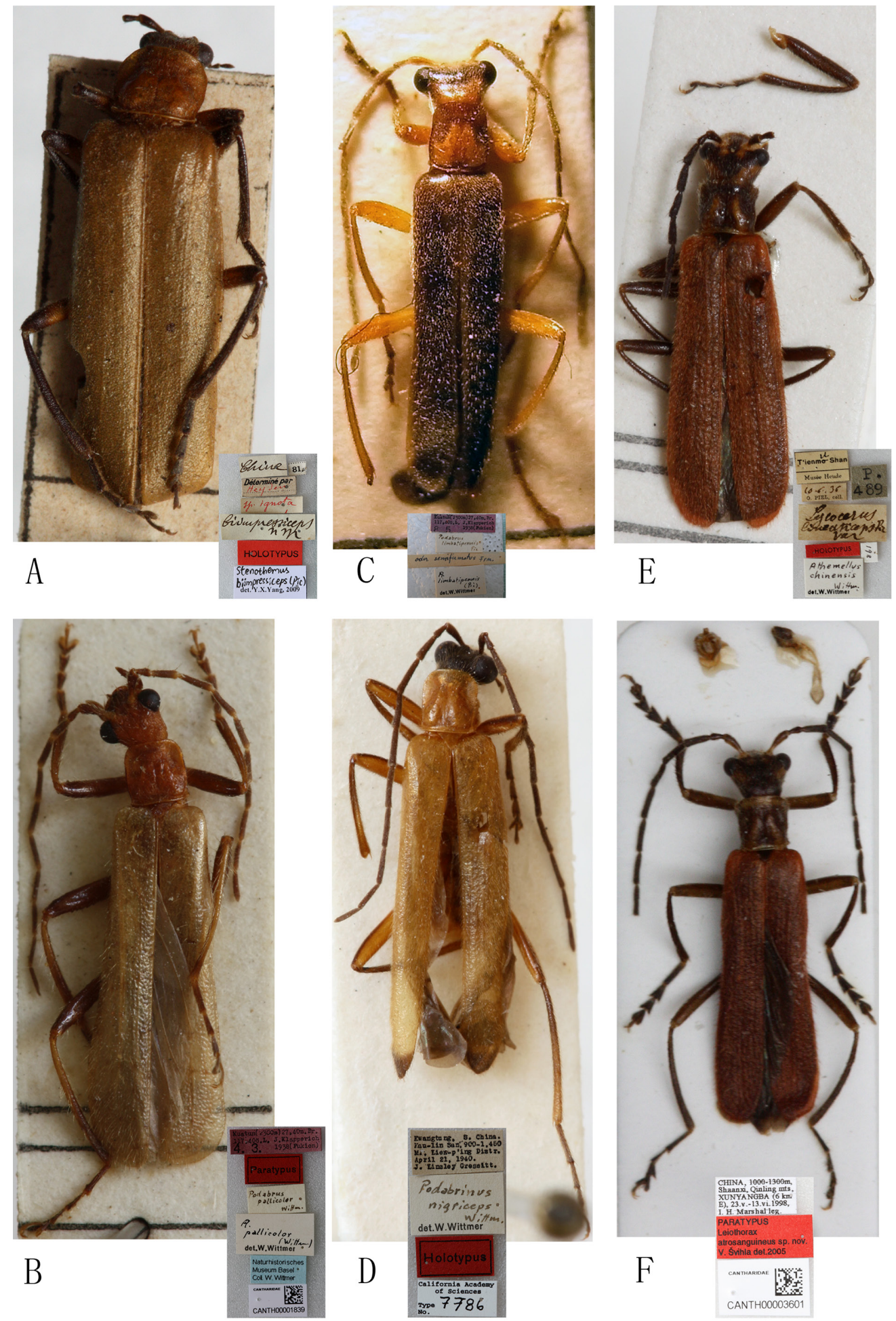

Fig. 1. Habitus, dorsal view. A. Stenothemus biimpressiceps (Pic, 1930) comb. nov., holotype, $q$ (MNHN), originally in Cantharis Linnaeus, 1758. B. S. pallicolor (Wittmer, 1951) comb. nov., paratype, $\widehat{\partial}$ (NHMB, CANTH00001839), originally in Podabrinus Fairmaire, 1896. C. S. limbatipennis (Pic, 1926) stat. rev. et comb. nov., ô, specimen determined by W. Wittmer (NHMB), originally in Podabrus. D. S. nigriceps (Wittmer, 1955) comb. nov., holotype, Ô (CAS, 7786), originally in Podabrinus. E. S. chinensis (Wittmer, 1982) comb. nov., holotype, o (MNHN), originally in Athemellus. F. Leiothorax atrosanguineus Švihla, 2005 syn. nov. of S. chinensis, holotype, ô (NHMB, CANTH00003601). 

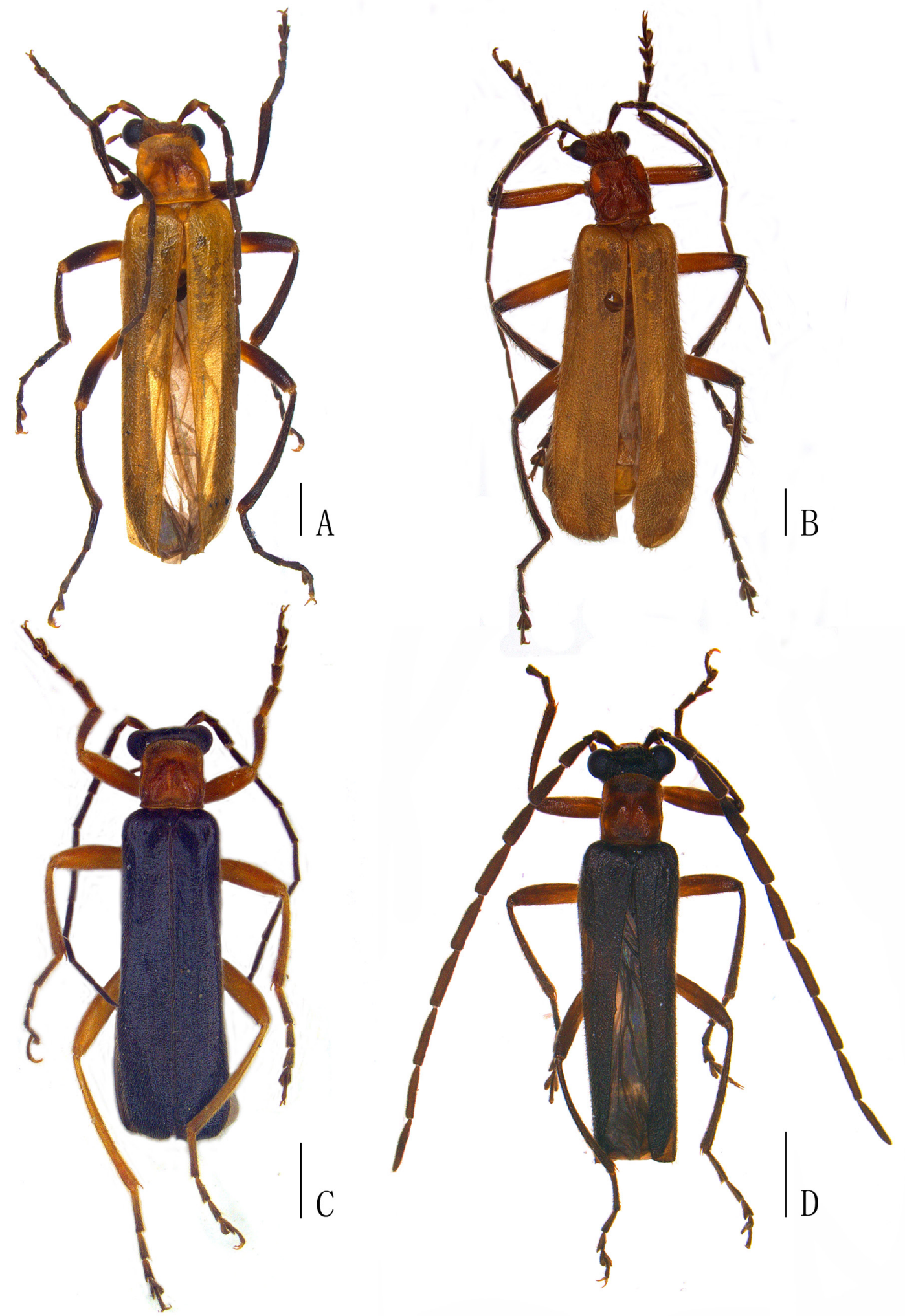

Fig. 2. Male habitus, dorsal view. A. Stenothemus biimpressiceps (Pic, 1930) comb. nov., nontype specimen from Zhejiang (IZAS). B. S. flavus Y. Yang \& X. Yang sp. nov., holotype, đ̊ (IZAS, IOZ(E)1123159). C. S. limbatipennis (Pic, 1926) stat. rev. et comb. nov., non-type specimen from Zhejiang (MHBU). D. S. longicornis Y. Yang \& H. Liu sp. nov., holotype, § (MHBU, HBU(E)410001). Scale bars: $1.0 \mathrm{~mm}$. 
Aedeagus (Fig. 3A-C). Strongly swollen laterally at basal part, shrunk apically in diameter; basal piece nearly as long as dorsal plate of each paramere, with a large, bifurcate conjoint middle nodule at base of ventral side; ventral process of each paramere nearly parallel to each other, short and distinctly thickened apically, bent dorsally in lateral view; dorsal plate slightly longer than ventral process, abruptly narrowed in middle and wide-triangularly protuberant at apical part, near to each other at basal part, apex acute and folded ventrally; apical parts of laterophyses depressed and separate from each other, apex acute and bent dorsally, not reaching middle emargination between dorsal plates.

Female (Fig. 1A)

Similar to male, but eyes less protruding, antennae shorter and roughly extending to elytral mid-length; pronotum slightly wider than long, elytra about 3.0 times as long as humeral width.

AbDOMINAL STERNITE VIII (Fig. 8A). Obliquely narrowed posteriorly, latero-apical angles obtusely rectangular, posterior margin deeply and roundly emarginate in middle and feebly sinuate on both sides, behind notch with membrane which presents small longitudinal sclerite at center.

INTERNAL ORGAN OF REPRODUCTIVE SYSTEM (Fig. 6A). Vagina stout, with median oviduct situated at ventroapical part, diverticulum and spermathecal duct arising from apex; diverticulum moderately long, about 0.12 times body length, evenly thinned apically, slender tube-shaped and spiral; spermathecal duct much shorter than diverticulum; spermatheca slender tube-shaped and spiral, obviously thinner than spermathecal duct and slightly longer than diverticulum, with basal part extended into short tube, where accessory gland opening. Accessory gland thin and obviously shorter than spermatheca.

\section{Remarks}

This species was originally described based on a single female type (Pic 1930). During our study, the type was located at MNHN, as well as some additional specimens, making it possible for us to study this species in detail. The morphological characters, especially the structure of the aedeagus, show that it belongs to Stenothemus and not to Cantharis.

\section{Distribution}

China (Anhui, Hubei, Zhejiang, Jiangxi).

Stenothemus pallicolor (Wittmer, 1951) comb. nov. Figs 1B, 3D-F, 6B, 8B

Podabrinus pallicolor Wittmer, 1951: 98.

Pseudoabsidia pallicolor - Wittmer 1969: 128.

Athemellus pallicolor-Wittmer 1972: 124.

Lycocerus pallicolor - Kazantsev \& Brancucci 2007: 252.

\section{Material examined}

\section{Holotype}

CHINA - Ô; [p] "Kuatun (2300m) 27, 40n. Br.//117. 40ö. L. J. Klapperich//1. 3.1938 (Fukien)", [h] "Podabrus//pallicolor//Wittm.", [h-p] "Holotypus//Podabrinus//pallicolor//n. sp.//Wittmer, 1949"; ZFMK.

\section{Paratype}

CHINA • 1 §’; [p] "Kuatun (2300m) 27, 40n. Br.// 117. 40ö. L. J. Klapperich // 4. 3.1938 (Fukien)”, [p] "Paratypus",[h]"Podabrinus // pallicolor//Wittm.",[h-p]"A.// pallicolor// (Wittmer.)//det.W.Wittmer", 

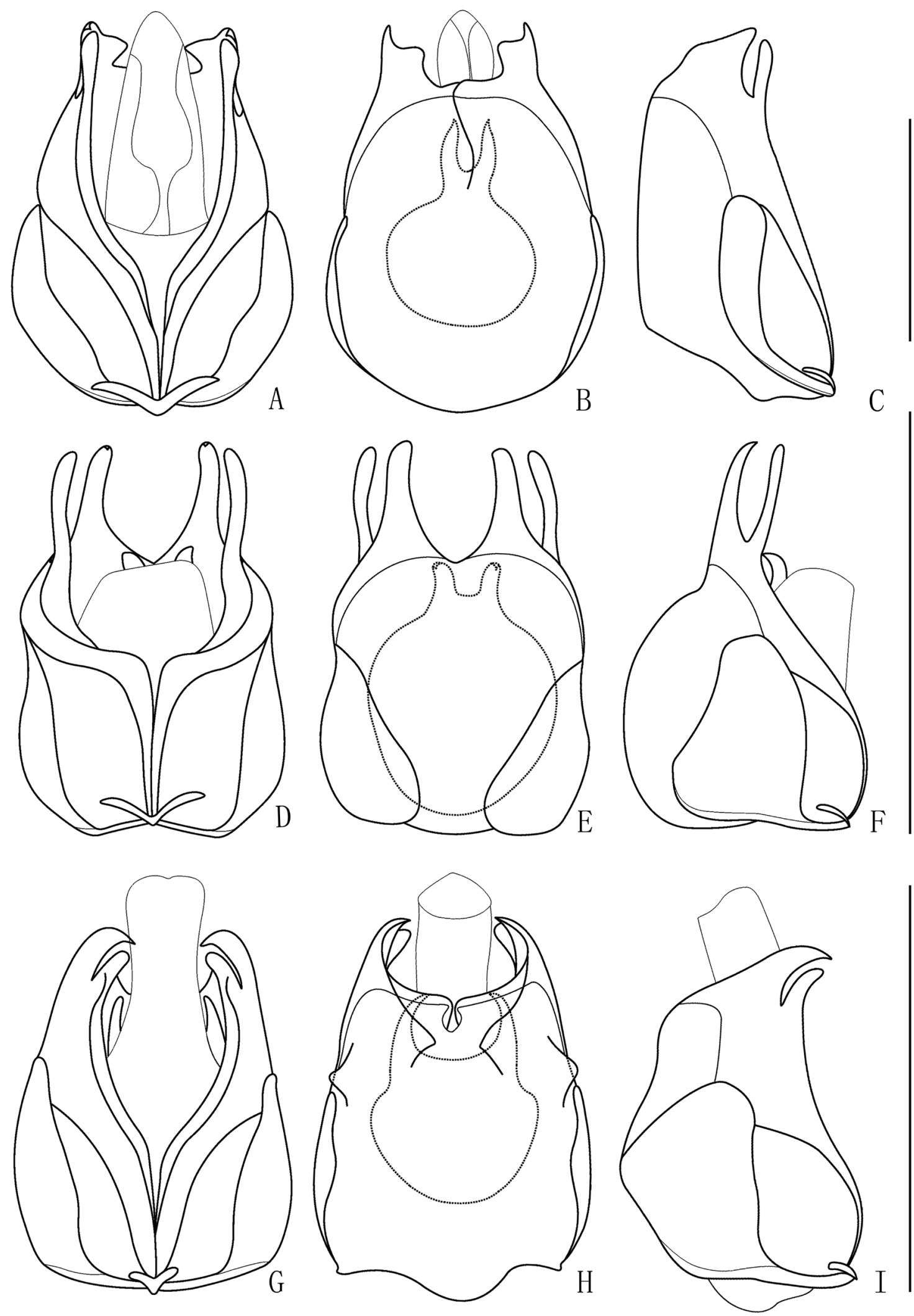

Fig. 3. Aedeagus (A, D, G = ventral view; B, E, H = dorsal view; $\mathrm{C}, \mathrm{F}, \mathrm{I}=$ lateral view). A-C. Stenothemus biimpressiceps (Pic, 1930) comb. nov. (IZAS). D-F. S. pallicolor (Wittmer, 1951) comb. nov. (IZAS). G-I. S. limbatipennis (Pic, 1926) stat. rev. et comb. nov. (MHBU). Scale bars: $1.0 \mathrm{~mm}$. 
[p] “Naturhistorisches // MuseumBasel//Coll.W.Wittmer",[p] “CANTHARIDAE// CANTH00001839”; NHMB.

\section{Other material}

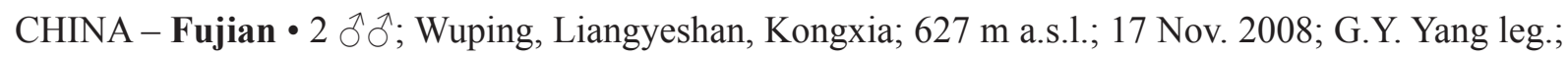


1946; Tschung Sen leg.; NHMB.

\section{Redescription}

MeAsurements. Body length (both sexes): 7.2-10.0 mm; width: 1.3-2.2 mm.

\section{Male (Fig. 1B)}

Coloration. Body dark brown, head, prothorax and scutellum testaceous, pronotum orange at anterior angles, elytra light brown, with irregular brown markings. Body mixed with short, recumbent and slightly long, semi-erect light yellow pubescence.

HEAD. Subquadrate, surface sparsely and finely punctate, each side with smooth and rectangular impression behind antennal socket; eyes strongly protruding, head width across eyes 1.2 times as wide as pronotum; terminal maxillary palpomeres long-triangular, widest in basal third; antennae filiform, extending to two-thirds length of elytra; antennomere II about twice as long as wide at apices, III about 2.5 times as long as II, IV longest, XI slightly longer than X, pointed at apex.

Pronotum. Subquadrate, about 1.1 times as long as wide, anterior margin nearly straight, anterior angles roundly truncate, lateral margins slightly sinuate, posterior margin straight and narrowly bordered, posterior angles nearly rectangular and slightly protruding, disc distinctly convex on posterolateral parts, surface finely and sparsely punctate.

ELYTRA. Slightly dilated posteriorly, about 4.3 times as long as humeral width, 6.0 times as long as pronotum, surface densely and finely punctate, present with hardly visible longitudinal costae.

Aedeagus (Fig. 3D-F). Strongly swollen dorsally at basal part, nearly parallel-sided in apical part; basal piece obviously as long as dorsal plate of each paramere, with a large, bifurcate conjoint middle nodule at base of ventral side; ventral process of each paramere nearly parallel to each another, long and slightly thickened apically, bent dorsally in lateral view; dorsal plate nearly as long as ventral process, strongly narrowed and even in width along the whole length, acute at apex and folded ventrally; apical parts of laterophyses depressed and separate from each other, apex acute and almost reaching middle emargination between dorsal plates.

\section{Female}

Similar to male, but body stouter, eyes less protruding, head width across eyes slightly wider than anterior margin of pronotum; antennae shorter, extending to elytral mid-length; elytra about 4.0 times as long as humeral width.

ABDOMinal STERNite VIII (Fig. 8B). Strongly narrowed posteriorly, latero-apical angles obtusely widetriangular, posterior margin deeply and rectangularly emarginate in middle and hardly emarginate on both sides, behind notch with membrane which presents small traverse-triangular sclerite at center.

INTERNAL ORGAN OF REPRODUCTIVE SYSTEM (Fig. 6B). Vagina stout, with median oviduct situated at ventroapical part, diverticulum and spermathecal duct arising from apex; diverticulum moderately long, about 0.12 times body length, evenly thinned apically, slender tube-shaped and spiral; spermathecal duct long but shorter than diverticulum; spermatheca slender tube-shaped and spiral, slightly thinner 
than spermathecal duct and shorter than diverticulum, with basal part extended into short tube, where accessory gland opening. Accessory gland thin, and shorter than spermatheca.

\section{Remarks}

The morphological characters, including the aedeagus, indicate that this species should belong to Stenothemus.

\section{Distribution}

China (Fujian).

Stenothemus limbatipennis (Pic, 1926) stat. rev. et comb. nov.

Figs $1 \mathrm{C}, 2 \mathrm{C}, 3 \mathrm{G}-\mathrm{I}, 6 \mathrm{C}, 8 \mathrm{C}$

Podabrus limbatipennis Pic, 1926: 235.

Lycocerus limatus Kazantsev, 2007: 54 (replacement name for Lycocerus limbatipennis (Pic, 1926), preoccupied by Pic, 1906). Syn. nov.

Pseudoabsidia limbatipennis - Wittmer 1969: 129.

Athemellus limbatipennis - Wittmer 1972: 124.

Lycocerus limbatipennis - Kazantsev \& Brancucci 2007: 251.

\section{Material examined}

\section{Determined material}

CHINA - Fujian • 1 đ̊; [p] "Kuatun (2300m) 27, 40n. Br.//117. 40ö. L. J. Klapperich//8. 5. 1938 (Fukien)", [h] "Podabrus//limbatipennis//Pic", [h] "odus. semifumatus. frm.", [h-p] "A.//limbatipennis//(Pic).// det. W. Wittmer"; NHMB • 1 ô; "Kuatun, 1946.V.2, leg, Tschung Sen"; NHMB • 1 q; Kuatun; 12 May 1946; Tschung Sen leg.; NHMB • 2 ổ; Shaowu, Tachulan; 6 May 1943; T.C. Maa leg.; NHMB.

\section{Other material}

CHINA - Fujian • 1 क; Chong'an, Xingcun, Guadun 8400-1160 m a.s.1.; 11 Apr. 1960; Y.R. Zhang leg.;

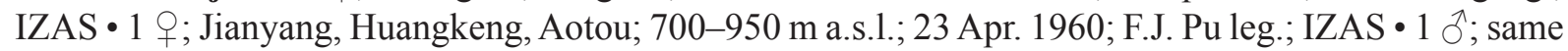
locality as for preceding; 800-1050 m a.s.1.; 26 Apr. 1960; Y.R. Zhang leg.; IZAS • $1{ }^{\text {त}}$; same locality as for preceding; 800-950 m a.s.l.; 29 Apr. 1960; Y.R. Zhang leg.; IZAS • 1 q; same locality as for preceding; 720-950 m a.s.1.; 30 Apr. 1960; C.L. Ma leg.; IZAS • 1 §̊; Wuyishan, San'gang; 740 m a.s.l.; Y.Y. Wu leg.; IZAS. - Jiangxi • 1 đ; Jinggangshan; 21 Apr. 2006; Y.B. Ba leg.; MHBU. - Zhejiang •

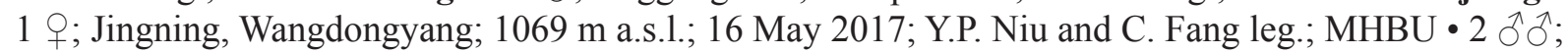
same locality and collectors as for preceding; $1150 \mathrm{~m}$ a.s.1.; 17 May 2017; MHBU • 2 ô $\widehat{\partial}, 4$ 우 ; same locality and collectors as for preceding; $970 \mathrm{~m}$ a.s.1.; 18 May 2017; MHBU $\bullet 2 \hat{\jmath}, 3$ 우; Chuanghua, Qingliangfeng, Longtansi; 14 May 2017; Y.B. Ba and H.H. Guan leg.; MHBU • 5 우; same locality as for preceding; 15-18 May 2012; J.S. Xu and L.X. Chang leg.; MHBU • 2 q $ᄋ$; same locality as for preceding; 20 May 2011; G.L. Xie leg.; MHBU • 1 §; Qingliangfeng, Tianchi; $21-23$ May 2012; J.S.

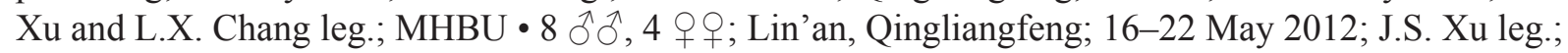
MHBU • $2 \hat{\jmath}, 1$; ; same collection data as for preceding; G.L. Xie leg.; MHBU • 9 q $q$; Qingliangfeng, Shunxiwu; 15-18 May 2012; J.S. Xu and L.X. Chang leg.; MHBU • 1 $\delta, 2$, $q$; same collection data as for preceding; G.L. Xie leg.; MHBU • 1 J, 2 우; Qingyuan, Baishanzu; 1557 m a.s.l.; 22 May 2017; Y.P. Niu and C. Fang leg.; MHBU $\bullet 1$ के; same locality and collectors as for preceding; 23 May 2017; MHBU • 1 đ̊; Pan'an, Dapanshan; 970 m a.s.1.; 13 May 2017; Y.P. Niu and C. Fang leg.; MHBU • 1 \%; same locality and collectors as for preceding; $641 \mathrm{~m}$ a.s.1.; 14 May 2017; MHBU • 2 क $\%$; Jingning, Dayanghu; 1030 m a.s.1.; 19 May 2017; Y.P. Niu and C. Fang leg.; MHBU. 


\section{Redescription}

MEAsurements. Body length (both sexes): 5.5-10.5 mm; width: 1.0-2.2 mm.

Male (Figs 1C, 2C)

Coloration. Body black, clypeus, mouthparts, antennomeres I and II, prothorax, legs except tarsi, posterior margins of abdominal ventrites and the entire terminal one orange, elytra light yellow at margins. Body densely covered with short, recumbent yellow pubescence.

HEAD. Subquadrate, surface densely and finely punctate, each side with smooth rectangular impression behind antennal socket; eyes moderately protruding, head width across eyes 1.3 times as wide as pronotum; terminal maxillary palpomeres long-triangular, widest in middle; antennae filiform, extending to two-thirds length of elytra, antennomere II about twice as long as wide at apices, III about 2.5 times as long as II, IV-XI each with smooth longitudinal impression in middle of outer edge, VI longest, $\mathrm{XI}$ slightly longer than $\mathrm{X}$, pointed at apex.

Pronotum. Subquadrate, about 1.1 times as long as wide, anterior margin slightly arcuate, anterior angles obtuse, subrounded, lateral margins nearly parallel and slightly sinuate, posterior margin straight, posterior angles moderately sharp and protruding, disc strongly convex on posterolateral parts, surface lustrous, finely and densely punctate.

ELYTRA. Parallel-sided, about 3.4 times as long as humeral width, 4.8 times as long as pronotum, humeral lustrous, the rest of surface densely and finely punctuate, with hardly indicated longitudinal costae.

Aedeagus (Fig. 3G-I). Moderately swollen laterally at basal part, shrunk apically in diameter; basal piece nearly as long as dorsal plate of each paramere, with a moderately large, bifurcate conjoint of? middle nodule at base of ventral side; ventral process of each paramere approaching to each other, short and slightly narrowed apically, bent ventrally in lateral view; dorsal plate slightly longer than ventral process, abruptly narrowed near base and bent to each other, apex acute and directing ventrally; apical part of laterophyses depressed and separated far from each other, apex acute and reaching middle emargination between dorsal plates.

\section{Female}

Similar to male, but more robust, eyes smaller, slightly protruding, antennae thinner and shorter, roughly extending to elytral mid-length, IV-XI without smooth longitudinal impressions; pronotum slightly wider.

ABDOMINAL STERNITE VIII (Fig. 8C). Strongly narrowed posteriorly, latero-apical angles widely rounded, posterior margin widely and roundly emarginate in middle and feebly sinuate on both sides, behind notch with membrane which is weakly sclerotised and tapered at apex.

INTERNAL ORGAN OF REPRODUCTIVE SYSTEM (Fig. 6C). Vagina elongate, with median oviduct situated at ventro-apical part, diverticulum and spermathecal duct arising from apex; diverticulum relatively short, about 0.08 times body length, evenly thinned apically, slender tube-shaped and spiral; spermathecal duct moderately long but obviously shorter than diverticulum; spermatheca slender tube-shaped and spiral, obviously thinner than spermathecal duct and extremely long, much longer than diverticulum, basal part extended into a moderately long tube, where accessory gland opening. Accessory gland moderately thin and shorter than spermatheca.

\section{Variation}

Head sometimes orange at vertex, pronotum more or less darkened at anterior part or both sides, elytra light yellow at more or less parts of margins. 


\section{Remarks}

The type was not located in MNHN although we tried several times; several specimens with labels written by Dr W. Wittmer with the specific name were found in NHMB. According to the previous work done by Wittmer $(1969,1972)$, it is speculated that he had examined the types during his working period, so the specimens determined by him are our references for the identification.

Kazantsev (2007) introduced the new name Lycocerus limatus for L. limbatipennis (Pic, 1926) (originally in Podabrus), which was preoccupied by Lycocerus limbatipennis (Pic, 1906) (originally in Cantharis). Judging from the male genitalia, Podabrus limbatipennis Pic, 1926 is a member of Stenothemus, to which it is transferred here. With the change of the genus, the replacement name Lycocerus limatus Kazantsev, 2007 becomes a junior synonym of S. limbatipennis (Pic, 1926) (ICZN Article 59.4).

\section{Distribution}

China (Zhejiang, Fujian, Jiangxi).

Stenothemus nigriceps (Wittmer, 1955) comb. nov.

Figs $1 \mathrm{D}, 4 \mathrm{~A}-\mathrm{C}, 6 \mathrm{D}, 8 \mathrm{D}$

Podabrinus nigriceps Wittmer, 1955: 39, fig. 1.

Pseudoabsidia nigriceps - Wittmer 1969: 128.

Athemellus nigriceps - Wittmer 1972: 124.

Lycocerus nigriceps - Kazantsev \& Brancucci 2007: 252.

\section{Material examined}

\section{Holotype}

CHINA - O’; [p] "Kwangtung. S. China.//Kau- lin San. 900-1.450//Mt. Lien-p'ing Distr.//April 21. 1940.//J. Linsley Gressitt”, [h-p] “Podabrinus // nigriceps //Wittm.// det. W. Wittmer”, [p] “Holotypus”, [p-h] "California Academy// of Sciences// Type//NO.7786"; CAS.

\section{Other material}

CHINA - Hong Kong • 1 q; “Taipokan, 2011.III.11, coll. Paul Aston”; MHBU • 1 § ; “Taipokan, 2014. II.13, coll. Paul Aston"; MHBU.

\section{Redescription}

MeAsurements. Body length (both sexes): 8.5-11.0 mm; width: 2.0-2.4 mm.

Male (Fig. 1D)

Coloration. Body yellow, head and apices of elytra black, antennae, tarsi and bases of tibiae dark brown. Body densely covered with short, recumbent yellow pubescence, mixed with slightly longer semi-erect pubescence on elytra.

HEAD. Subquadrate, surface densely and finely punctuate, each side with smooth rectangular impression behind antennal socket; eyes strongly protruding, head width across eyes about 1.2 times as wide as pronotum; terminal maxillary palpomeres long-triangular, widest in middle; antennae filiform, extending to two-thirds length of elytra; antennomeres II about 2.5 times as long as wide at apices, III about twice as long as II, IV longest, XI slightly longer than X, pointed at apex. 

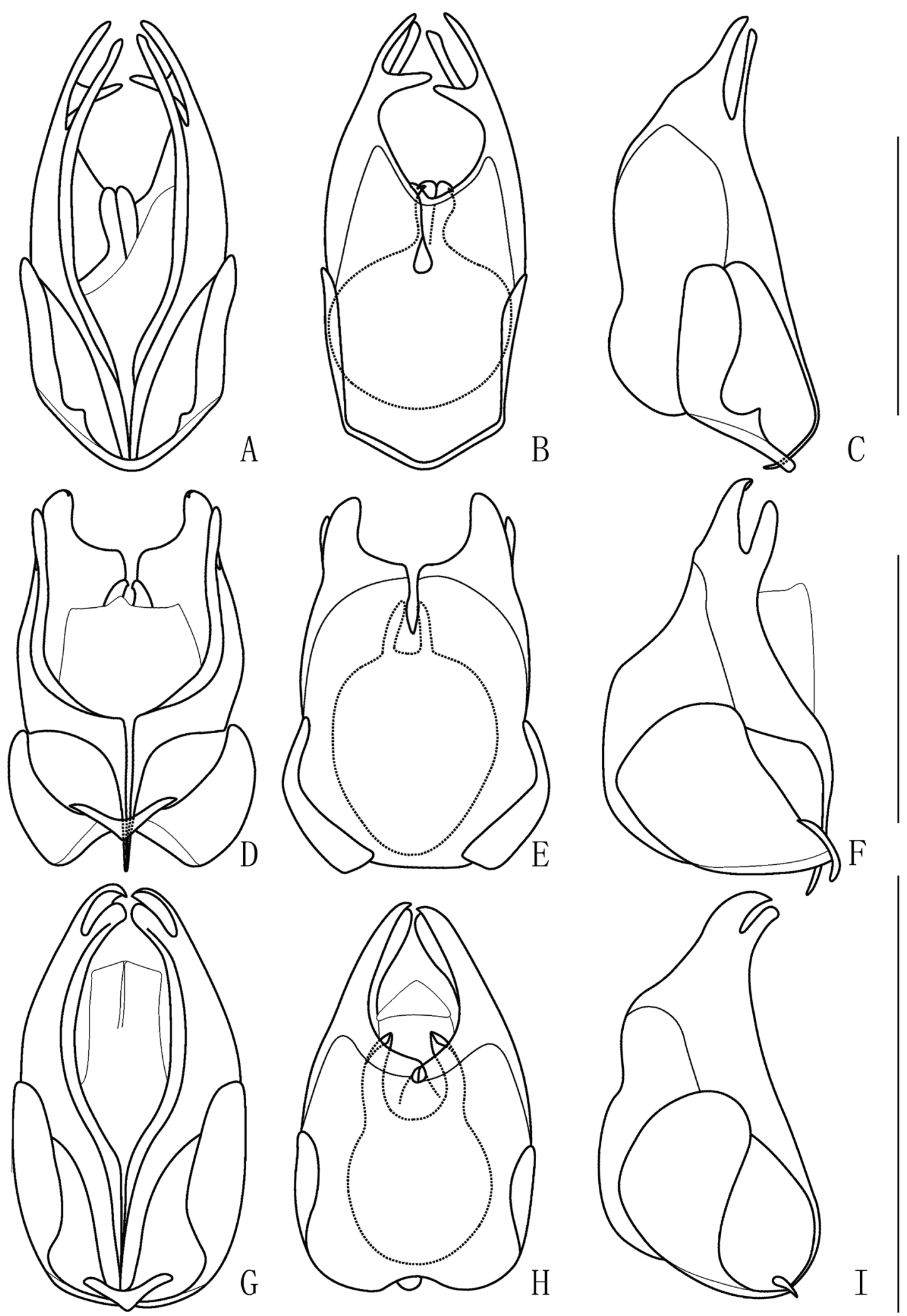

Fig. 4. Aedeagus (A, D, G = ventral view; $\mathrm{B}, \mathrm{E}, \mathrm{H}=$ dorsal view; $\mathrm{C}, \mathrm{F}, \mathrm{I}=$ lateral view). A-C. Stenothemus nigriceps (Wittmer, 1955) comb. nov. (MHBU). D-F. S. flavus Y. Yang \& X. Yang sp. nov. (IZAS, IOZ(E)1123159). G-I. S. longicornis Y. Yang \& H. Liu sp. nov. (MHBU, HBU(E)410001). Scale bars: $1.0 \mathrm{~mm}$ 
Pronotum. Subquadrate, about 1.2 times as long as wide, anterior margin nearly arcuate, anterior angles widely rounded, lateral margins nearly parallel, posterior margin straight, posterior angles moderately sharp, protruding, disc distinctly convex on posterolateral parts, surface finely and densely punctate.

ELYTRA. Parallel-sided, about 4.0 times as long as humeral width, 4.2 times as long as pronotum, surface densely and finely punctate, with hardly indicated longitudinal costae.

Aedeagus (Fig. 4A-C). Weakly swollen dorsally in basal part, weakly contracted in diameter basally; basal piece obviously shorter than dorsal plate of each paramere, each with triangular protuberance but without conjoint middle nodule at base of ventral side; ventral processes of parameres approaching to each other, long and slightly widened at apices, bent dorsally in lateral view; dorsal plate slightly longer than ventral process, strongly narrowed apically, narrow-triangularly protuberant in middle, apex acute and approaching to each other; apical part of laterophyses compressed and next to each other in middle, apex hooked and directed laterally, slightly exceeding middle emargination between dorsal plates.

\section{Female}

Similar to male, but body stouter, eyes less protruding, head width across eyes slightly wider than anterior margin of pronotum; antennae narrower and shorter, extending to elytral mid-length; pronotum wider, nearly as long as wide; elytra slightly dilated posteriorly, about 3.1 times as long as humeral width.

AbDominal SteRnite VIII (Fig. 8D). Obliquely narrowed posteriorly, latero-apical angles widely triangular, posterior margin deeply and roundly emarginate in middle and nearly straight on both sides (membrane missing behind notch).

INTERNAL ORGAN OF REPRODUCTIVE SYSTEM (Fig. 6D). Vagina moderately elongate, with median oviduct situated at ventro-apical part, diverticulum and spermathecal duct arising from apex; diverticulum moderately long, about 0.16 times body length, evenly thinned apically, slender tube-shaped and spiral; spermathecal duct much shorter than diverticulum; spermatheca slender tube-shaped and spiral, obviously thinner than spermathecal duct and shorter than diverticulum, with basal part extended into short tube, where accessory gland opening. Accessory gland thin and shorter than spermatheca.

\section{Remarks}

In this study, the structure of abdominal sternite VIII of the female is incomplete. Because there is only one female specimen, the structural description of abdominal sternite VIII needs to be improved.

\section{Distribution}

China (Guangdong, Hong Kong).

Stenothemus chinensis (Wittmer, 1982) comb. nov.

Figs 1E-F, 5G-I, 7C, 8G

Athemellus chinensis Wittmer, 1982: 344, fig. 1.

Leiothorax atrosanguineus Švihla, 2005: 104, figs 72-74. Syn. nov.

Lycocerus chinensis - Kazantsev \& Brancucci 2007: 250.

\section{Material examined}

\section{Holotype of Athemellus chinensis}

CHINA • O; [h-p] "u//T" ienmo-Shan//Musée Heude", [h-p] "10. 6. 36//O. PIEI, coll.", [p] "P.//489", [h] "Lycocerus//lineaticeps Pic//n. sp.", [p] "HOLOTYUS", [h]"198", [h-p] "Athemellus// chinensis//Wittmer.// det. W. Wittmer"; MNHN. 
Holotype of Leiothorax atrosanguineus

CHINA • O ; [p] "CHINA, 1000-1300m//Shaanxi, Qinling mts, //XUNYANGBA (6km//E), 23.v.-13. vi.1998, // I. H. Marshal leg.", [p] "HOLOTYPUS //Leiothorax// atrosanguineus sp. nov.//V. Švihla det. 2005"; "CANTHARIDAE//CANTH000036013"; NHMB.

\section{Other material}

CHINA - Fujian • 1 đ’; Chong'an, Xingcun, San'gang; 740 m a.s.1.; 25 May 1960; Y.R. Zhang leg.; IZAS 1 $1 \hat{\jmath}$; same collection data and collector as for preceding, 17 May 1960; IZAS. - Guangxi •

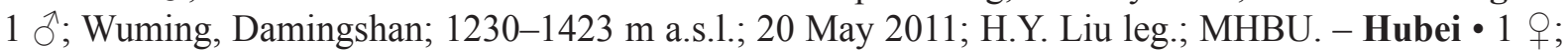
Xingshan, Longmenhe; 1300 m a.s.l.; 21 Jun. 1993; R.Z. Huang leg.; IZAS • 1 .; same locality as for preceding; 1280 m a.s.1.; 23 Jun. 1993; W.Z. Li leg.; IZAS • 1 क; Shennongjia; 19 Jul. 1980; P.Y. Yu leg.; IZAS • 1 q; Shennongjia, Hongping; 1660 m a.s.1.; 16 Jul. 1981 Y.H. Han leg.; IZAS • 1 ᄋ; same locality and collector as for preceding; 19 Jul. 1981; IZAS • 1 \%; same locality as for preceding; 6 Jun. 2018; P. Wang leg.; MHBU • 1 ô, 1 ๆ; Shennongjia, Hongping, Wenshui; 1 Jun. 2018; P. Wang and L.M. Li leg.; MHBU • 1 ऊ’; Shennongjia, Jiuchong; 711.9 m a.s.1.; 11-15 Jul. 2014; Y.B. Ba and S.Y. Tang leg.; MHBU • 1 q; Yichang, Dalaoling; 6 May 2015; T.F. Qiu leg.; MHBU • 1 क; Zhuxi, Xiangba; 1208 m a.s.1.; 5 Jul. 2017; Z.Z. Xia leg.; IZAS • 1 đ’; Shennongjia, Baozidong; 23 May 2018; P. Wang leg.; MHBU • 1 ơ; Dongxi management zone; 17 May 2019; P. Wang leg.; MHBU. Jiangxi • 1 q; Shangrao, Sanqingshan; 15-20 Apr. 2007; L. Shi leg.; SYSU. - Shaanxi • 1 q; Yangxian, Huayang, Yantoushang; 1206 m a.s.1.; 107³1'6.9" E, 33³8'29" N, 27 Jun. 2017; H.Y. Liu leg.; IZAS. - Sichuan • 1 क; Emeishan, Jiulaodong; 1800-1900 m a.s.l.; 13 Jun. 1957; Y.C. Lu leg.; IZAS • 1 O ; Emeishan; 1600-2100 m a.s.1.; 24 Jun. 1955; Бущик leg.; IZAS. - Yunnan・1 ðَ; Lushui, Yaojiaping; $2500 \mathrm{~m}$ a.s.l.; 24 May 1981; S.Y. Wang leg.; IZAS • 1 q; Zhibenshan; $2550 \mathrm{~m}$ a.s.1.; 22 Apr. 1981; S.Y. Wang leg.; IZAS. - Zhejiang • 1 §ं; Qingyuan, Baishanzu; 1500 m a.s.1.; 18 May 1994; H. Wu leg.;

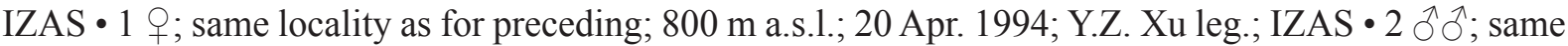
collection data as for preceding; leg. H. Wu; IZAS • 1 万; Tianmushan, Bingchuan Valley; 7 Jun. 2011; H.T. Song leg.; MHBU • 1 क; Changhua, Jiufu; 13 May 2017; Y.B. Ba and H.H. Guan leg.; MHBU


Musée Heude, 1936.VI.10; leg. O. Piel”; IZAS.

\section{Supplementary description}

MEASUREMENTs. Body length (both sexes): $7.0-10.5 \mathrm{~mm}$; width: $1.2-2.3 \mathrm{~mm}$.

\section{Male (Fig. 1F)}

Aedeagus (Fig. 5G-I). Moderately swollen laterally at basal part, contracted apically in diameter; basal piece nearly as long as dorsal plate of each paramere, with a moderately large, parallel-sided conjoint middle nodule at base of ventral side; ventral processes of parameres nearly parallel to each other, short and slightly narrowed apically, bent dorsally in lateral view; dorsal plate slightly shorter than ventral process, evenly narrowed apically, apex acute and bent to each other; apical part of laterophyses compressed and next to each other in middle, dorsolateral margin emarginate near apex, rounded and obviously exceeds middle emargination between dorsal plates.

Female (Fig. 1E)

ABDOMINAL STERNITE VIII (Fig. 8G). Obliquely narrowed posteriorly, latero-apical angles obtusely rectangular, posterior margin deeply and triangularly emarginate in middle and feebly sinuate on both sides, behind notch with membrane which weakly sclerotised and rounded at apex.

INTERNAL ORGAN OF REPRODUCTIVE SYSTEM (Fig. 7C). Vagina stout, with median oviduct situated at ventroapical part, diverticulum and spermathecal duct arising from apex; diverticulum moderately long, about 0.12 times body length, evenly thinned apically, slender tube-shaped and spiral; spermathecal duct much shorter than diverticulum; spermatheca slender tube-shaped and spiral, obviously thinner 

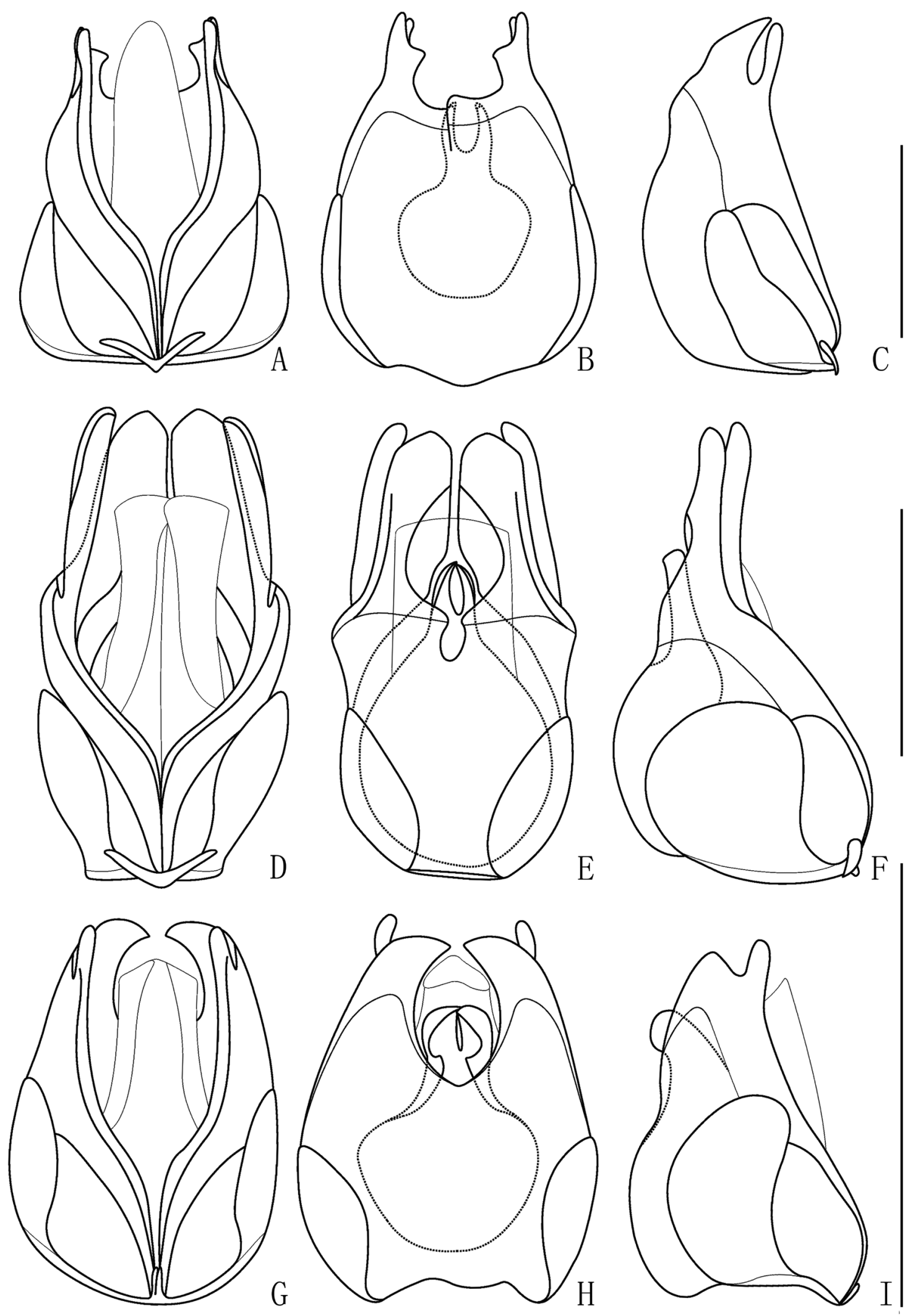

Fig. 5. Aedeagus (A, D, G = ventral view; B, E, H = dorsal view; C, F, I = lateral view). A-C. Stenothemus fukienensis Wittmer, 1974 (IZAS, IOZ(E)1659634). D-F. S. kuatunensis Wittmer, 1979 (IZAS). G-I. S. chinensis (Wittmer, 1982) comb. nov. (IZAS, IOZ(E)1436585). Scale bars: $1.0 \mathrm{~mm}$. 


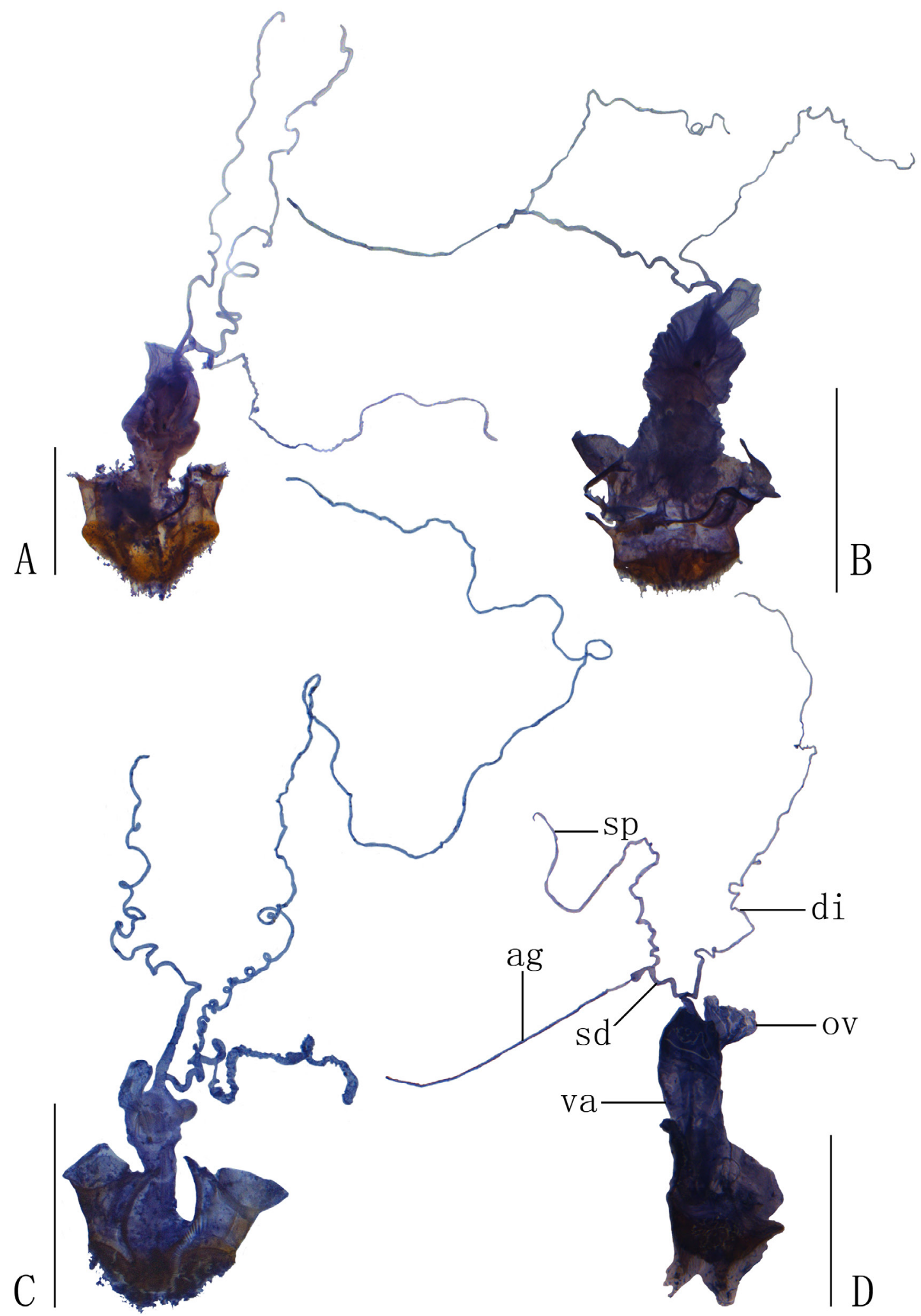

Fig. 6. Female internal reproductive system, lateral view. A. Stenothemus biimpressiceps (Pic, 1930) comb. nov. (IZAS, IOZ(E)1435863). B. S. pallicolor (Wittmer, 1951) comb. nov. (SYSU, En-076948). C. S. limbatipennis (Pic, 1926) stat. rev. et comb. nov. (MHBU). D. S. nigriceps (Wittmer, 1955) comb. nov. (MHBU). Scale bars: $0.5 \mathrm{~mm}$. 
than spermathecal duct and shorter than diverticulum, with basal part extended into short tube, where accessory gland opening. Accessory gland thin and much longer than spermatheca.

\section{Remarks}

Wittmer (1982), having only females at his disposal, assigned this species to the genus Athemellus Wittmer, 1972, now a junior synonym of Lycocerus Gorham, 1889 (Okushima 2005). Some additional material, also including males from the type locality, is available here, and the morphological characters show that this species is a member of Stenothemus.

Furthermore, the holotype male of Leiothorax atrosanguineus shows that it has no differences from S. chinensis comb. nov.; also, a large series of additional material from several localities is available for examination though their type localities are far away. Therefore, $L$. atrosanguineus is considered to be a junior synonym of $S$. chinensis.

\section{Distribution}

China (Shaanxi, Zhejiang, Hubei, Fujian, Sichuan, Jiangxi, Yunnan, Guangxi).

Stenothemus flavus Y. Yang \& X. Yang sp. nov.

urn:1sid:zoobank.org:act:0F33A6EA-1C0A-4528-8A44-CF59FC42EF73

Figs 2B, 4D-F, 7A, 8E

\section{Differential diagnosis}

The new species is related to S. melleus Švihla, 2005 (located in Sichuan, China), but can be distinguished by its larger body (no more than $9.0 \mathrm{~mm}$ length in the latter); femur orange, darkened only at base (in the latter, darkened at least at apical third part); aedeagus: middle emargination between dorsal plates deep, nearly as long as half its length (shallow in the latter, much shorter than half length).

\section{Etymology}

The specific name is derived from the Latin 'Alavus', meaning 'golden-yellow' and referring to its body coloration.

\section{Material examined}

\section{Holotype}

CHINA - O’; Zhejiang, Xitianmushan, Xianrending; 1500 m a.s.1.; 30 May 1998; H. Wu leg.; IZAS IOZ(E)1123159.

\section{Paratypes}

CHINA - Zhejiang • 1 ô; Anji, Longwangshan; $490 \mathrm{~m}$ a.s.1.; 17 Jul. 1995; H. Wu leg.; IZAS IOZ(E)1659904 - 1 क; same locality and collector as for preceding; 770 m a.s.1.; 19 Oct. 1995; IZAS IOZ(E)1659887 • 1 § ; same locality and collector as for preceding; 21 Oct. 1995; IZAS IOZ(E)1659896 • 1 ठ̊; Xitianmushan; 500 m a.s.1.; 21 Jul. 1998; M.S. Zhao leg.; IZAS IOZ(E)225767 • 1 đ̊; Xitianmushan, Chanyuansi; 380 m a.s.1.; 13 Nov. 1998; H. Wu leg.; IZAS IOZ(E)215768 • 1 o; same collection data as for preceding; IZAS IOZ(E)215769 • 1 \%; Tianmushan; 12 Nov. 1998; M.S. Zhao leg.; IZAS IOZ(E)215770 • 1 đ; Tianmushan; 24 Nov. 1998; C.L. Ma leg.; IZAS IOZ(E)215771 • 1 Ј ; Qingyuan, Baishanzu; 1500 m a.s.l.; 23 Oct. 1993; H. Wu leg.; IZAS IOZ(E)215772. 


\section{Description}

Measurements. Body length (both sexes): $9.0-14.0 \mathrm{~mm}$ (12.0 mm in holotype); width: $2.5-3.0 \mathrm{~mm}$ (2.8 $\mathrm{mm}$ in holotype).

Male (Fig. 2B)

Coloration. Body yellow, head and prothorax testaceous, pronotum orange at anterior angles, mouthparts, antennae, tarsi, tibiae and bases of femora black brown. Body densely mixed with short recumbent and slightly longer semi-erect light yellow pubescence.

HEAD. Subquadrate, surface sparsely and finely punctate, each side with smooth and rectangular impression behind antennal socket; eyes strongly protruding, head width across eyes 1.2 times as wide as pronotum; terminal maxillary palpomeres long-triangular, widest at basal third; antennae filiform, extending to two-thirds length of elytra, antennomere II about 2.3 times as long as wide at apices, III about 1.7 times as long as II, IV longest, XI slightly longer than X, pointed at apex.

Pronotum. Subquadrate, about 1.1 times as long as wide, anterior margin nearly straight, anterior angles roundly truncate, lateral margins slightly sinuate, posterior margin slightly sinuate, posterior angles moderately sharp and protruding, disc strongly convex on posterolateral parts, surface finely and densely punctate.

ElYTRA. Moderately dilated posteriorly, about 3.6 times as long as humeral width, 5.1 times as long as pronotum, surface densely and finely punctate, with hardly indicated longitudinal costae.

Aedeagus (Fig. 4D-F). Strongly swollen dorsally at basal part, nearly parallel-sided in apical part; basal piece nearly as long as dorsal plate of each paramere, with a large, bifurcate conjoint middle nodule at base of ventral side; ventral processes of parameres nearly parallel to each other, short and slightly narrowed apically, bent dorsally in lateral view; dorsal plate slightly longer than ventral process, abruptly narrowed in middle, near to each other at basal part, acute at apex and folded ventrally; apical part of laterophyses depressed and separate from one another, apex acute and not reaching middle emargination between dorsal plates.

\section{Female}

Similar to male, but stouter, eyes less protruding; antennae shorter, reaching elytral mid-length, pronotum wider, nearly as wide as long.

Abdominal sternite VIII (Fig. 8E). Moderately narrowed posteriorly, latero-apical angles widely rounded, posterior margin widely and rectangularly emarginate in middle and truncate on both sides, behind notch with membrane which sclerotised and triangularly bilobed at apex and present with traverse-triangular sclerite at center and small rounded sclerite on each side.

INTERNAL ORGAN OF REPRODUCTIVE SYSTEM (Fig. 7A). Vagina stout, with median oviduct situated at ventroapical part, diverticulum and spermathecal duct arising from apex; diverticulum moderately long, about 0.16 times body length, evenly thinned apically, slender tube-shaped and spiral; spermathecal duct longer than diverticulum; spermatheca slender tube-shaped and spiral, obviously thinner than spermathecal duct and much longer than diverticulum, with basal part extended into short tube, where accessory gland opening. Accessory gland thin and much shorter than spermatheca.

\section{Distribution}

China (Zhejiang). 


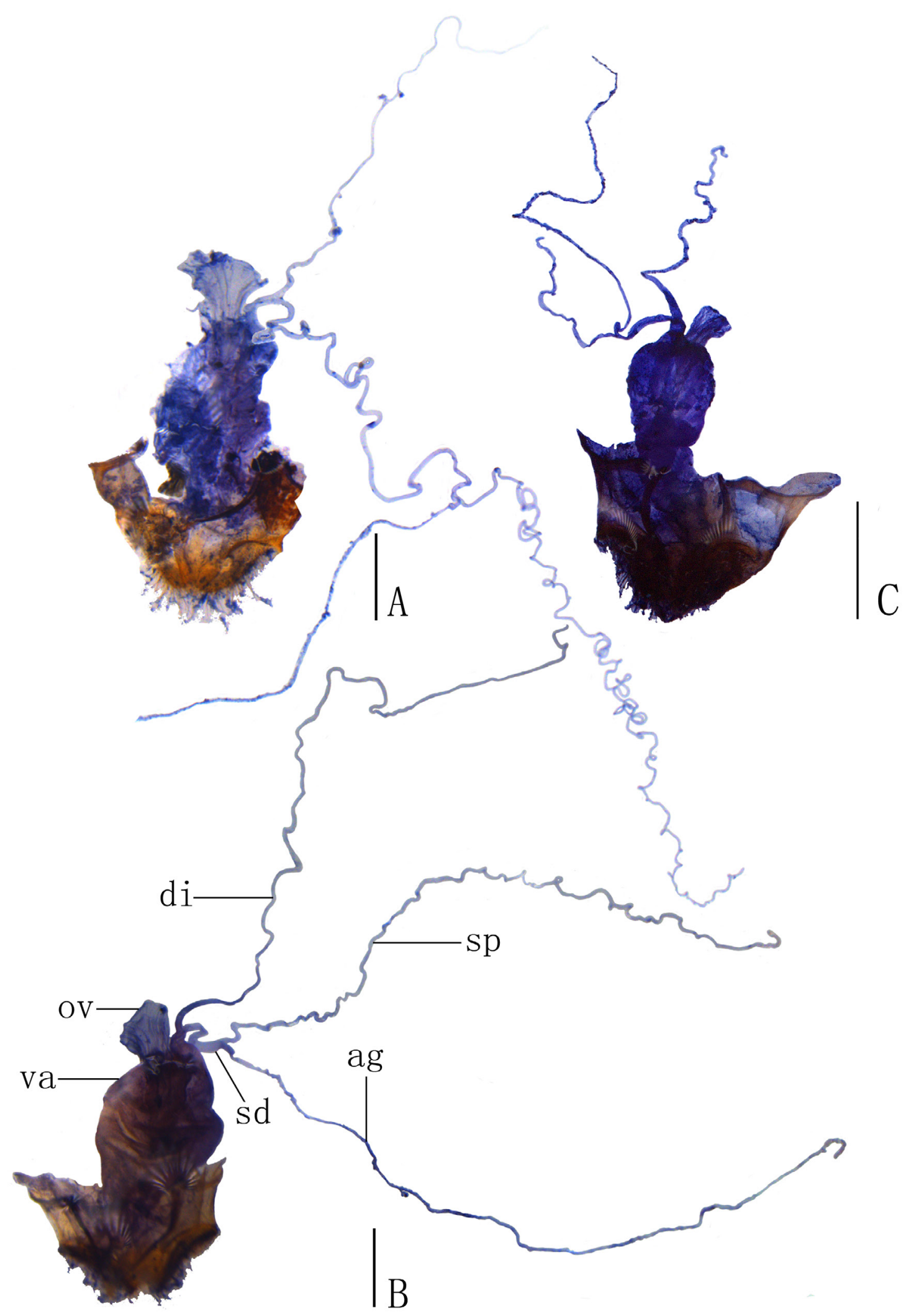

Fig. 7. Female internal reproductive system, lateral view. A. Stenothemus flavus Y. Yang \& X. Yang sp. nov. (IZAS, IOZ(E)1659887). B. S. fukienensis Wittmer, 1974 (IZAS). C. S. chinensis (Wittmer, 1982) comb. nov. (MHBU). Scale bars: $0.5 \mathrm{~mm}$. 


\section{Stenothemus longicornis Y. Yang \& H. Liu sp. nov. urn:1sid:zoobank.org:act:642A908A-1F99-44DD-BC37-142F864A35E2}

Figs 2D, 4G-I

\section{Differential diagnosis}

The new species resembles $S$. hajeki Švihla, 2011 (from N Yunnan, China), but differs in the following characters: antennae without impressions on middle antennomeres (with small oval impressions on antennomeres IV-XI in the latter), pronotum obviously longer than wide (nearly as long as wide in the latter); elytra more elongate, about 3.5 times humeral width (3.2 times in the latter); aedeagus: parameres approaching each other (far from each other in the latter).

\section{Etymology}

The specific name is derived from the Latin 'longus', meaning 'long', and 'cornus', meaning 'horn', and referring to its long antennae.

\section{Material examined}

\section{Holotype}

CHINA • ${ }^{\text {; }}$; Guangdong, Nanling, Ruyuan; 8 Apr. 2009; L. Gao leg.; MHBU HBU(E)410001.

\section{Description}

Male (Fig. 2D)

MEASUREMENTs. Body length: $6.8 \mathrm{~mm}$; width: $1.4 \mathrm{~mm}$.

Coloration. Body black, mouthparts light brown, reddish brown at apices of mandibles, more or less darkened at maxillary and labial palpi, prothorax reddish brown, femora light brown at basal. Body densely covered with short, recumbent yellow pubescence.

HEAD. Subquadrate, surface densely and finely punctate, each side with smooth and rectangular impression behind antennal socket; eyes strongly protruding, head width across eyes about 1.4 times as wide as pronotum; terminal maxillary palpomeres long-triangular, widest at basal two-thirds; antennae quite long, obviously exceeding apices of elytra; antennomeres II slightly longer than wide at apices, III about 2.6 times as long as II, III-XI flattened and subparallel-sided, IV widest, V to XI gradually narrowing towards apex, VIII longest, XI slightly longer than X, pointed at apex.

Pronotum. Subquadrate, about 1.2 times as long as wide, anterior margin rounded, anterior angles obtuse, subrounded, lateral margins nearly parallel and slightly sinuate, posterior margin straight and finely bordered, posterior angles obtusely rectangular, disc strongly convex on posterolateral parts, surface finely and densely punctate.

ELYTRA. Parallel-sided, about 3.5 times as long as humeral width, 4.8 times as long as pronotum, surface densely and finely punctate, with hardly visible longitudinal costae.

Aedeagus (Fig. 4G-I). Moderately swollen laterally at basal part, strongly contracted in diameter apically; basal piece nearly as long as dorsal plate of each paramere, with a large, bifurcate conjoint middle nodule at base of ventral side; ventral processes of parameres approaching each other, short and slightly thickened apically, bent ventrally in lateral view; dorsal plate slightly longer than ventral process, strongly narrowed near base, approaching to each other, apex acute and directed ventrally; apical part of laterophyses separate from each other, apex wide-triangular and bent dorsally, feebly exceeding middle emargination between dorsal plates. 


\author{
Female \\ Unknown.

\section{Distribution} \\ China (Guangdong).
}

\author{
Stenothemus fukienensis Wittmer, 1974 \\ Figs $5 \mathrm{~A}-\mathrm{C}, 7 \mathrm{~B}, 8 \mathrm{~F}$
}

Stenothemus fukienensis Wittmer, 1974: 60, figs 20-21.

\title{
Material examined
}

Holotype

CHINA • O’; [p] "Kuatun (2300m) 27, 40n. Br.//117. 40ö. L. J. Klapperich//3. 4. 1938 (Fukien)", [h] "Stenothemus//fukienensis//Wittm.//det. W. Wittmer", [p] "HOLOTYPUS", [p] "Naturhistorisches//Museum Basel//Coll. W. Wittmer", [p] "Cantharidae//CANTH00003399"; NHMB.

\section{Other material}

CHINA - Fujian • 1 đ̊; Shaowu, Tachuland; 14 May 1945; collector unknown; NHMB. - Zhejiang

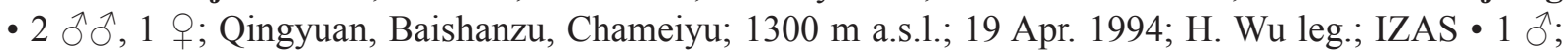
same locality as for preceding; $800 \mathrm{~m}$ a.s.l.; 20 Apr. 1994; W.G. Zhang leg.; IZAS • 1 §ं; Longquan, Fengyangshan; $1800 \mathrm{~m}$ a.s.l.; 18 May 2007; J.F. Gao and B.F. Zhou leg.; IZAS • 1 \%; same locality as for preceding; $1250 \mathrm{~m}$ a.s.1.; 10 May 2007; S.L. Liu leg.; IZAS.

\section{Supplementary description}

\section{Male}

Aedeagus (Fig. 5A-C). Strongly swollen laterally in basal part, contracted in diameter apically; basal piece nearly as long as dorsal plate of each paramere, with a large, bifurcate conjoint middle nodule at base of ventral side; ventral processes of parameres nearly parallel to one another, distinctly thickened apically, bent dorsally in lateral view; dorsal plate slightly longer than ventral process, abruptly narrowed in middle and wide-triangularly protuberant at apical part, near to each other at basal part, apex acute and folded ventrally; apical parts of laterophyses depressed and separate from each other, apex acute and bent dorsally, reaching middle emargination between dorsal plates.

\section{Female}

ABdOMINAL STERNITE VIII (Fig. 8F). Moderately narrowed posteriorly, posterior margin deeply and triangularly emarginate in middle and feebly emarginate on both sides; behind notch with membrane, narrowly bilobed at apex and present with longitudinal sclerite at center.

INTERNAL ORGAN OF REPRODUCTIVE SYSTEM (Fig. 7B). Vagina stout, with median oviduct situated at ventroapical part, diverticulum and spermathecal duct arising from apex; diverticulum extremely long, about 0.33 times body length, evenly thinned apically, slender tube-shaped and spiral; spermathecal duct much shorter than diverticulum; spermatheca slender tube-shaped and spiral, obviously thinner than spermathecal duct and nearly as long as diverticulum, with basal part extended into short tube, where accessory gland opening. Accessory gland thin and nearly as long as spermatheca.

\section{Remarks}

This species was described only based on the male, and the female is here recorded for the first time. 

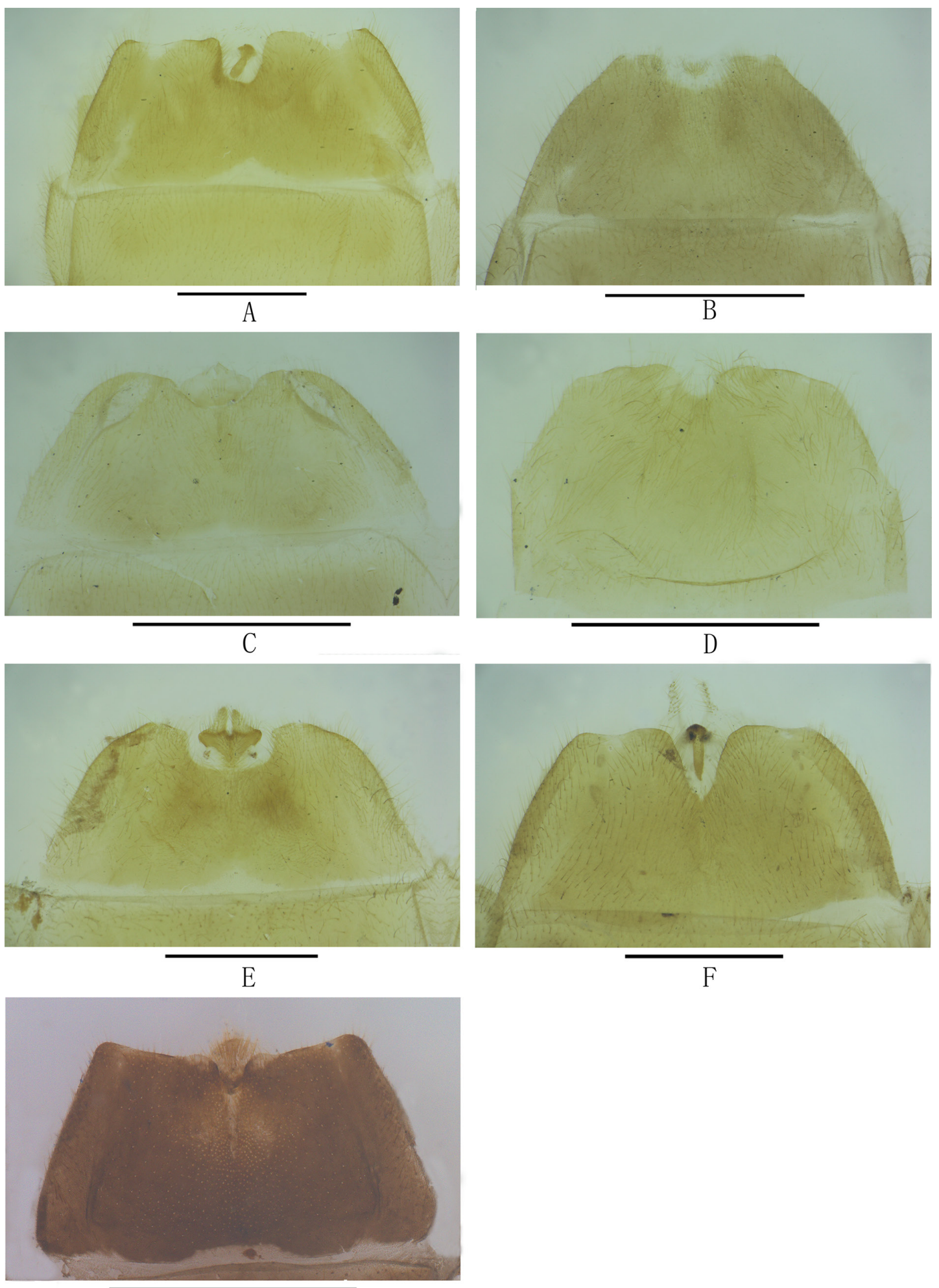

G

Fig. 8. Female abdominal sternite VIII, ventral view. A. Stenothemus biimpressiceps (Pic, 1930) comb. nov. (IZAS, IOZ(E)1435863). B. S. pallicolor (Wittmer, 1951) comb. nov. (SYSU, En-076948). C. S. limbatipennis (Pic, 1926) stat. rev. et comb. nov. (MHBU). D. S. nigriceps (Wittmer, 1955) comb. nov. (MHBU). E. S. flavus Y. Yang \& X. Yang sp. nov. (IZAS, IOZ(E)1659887). F. S. fukienensis Wittmer, 1974 (IZAS). G. S. chinensis (Wittmer, 1982) comb. nov. (MHBU). Scale bars: $1.0 \mathrm{~mm}$. 


\section{Distribution}

China (Zhejiang, Fujian).

Stenothemus kuatunensis Wittmer, 1979

Fig. 5D-F

Stenothemus kuatunensis Wittmer, 1979: 330, fig. 2.

\section{Material examined}

\section{Holotype}

CHINA • ô; [p] "KUATUN, FUKIEN//China, 26.9.(19)46//(TSCHUNG SEN.)", [h] "Stenothemus // kuatunensis//Wittm.", [p] "Naturhistorisches//Museum Basel//Coll. W. Wittmer", [p]“CANTHARIDAE//CANTH00002946”; NHMB.

\section{Other material}

CHINA - Anhui • 2 ððત; Fengyangshan, Miaopudi; 1430 m a.s.l.; 24 Aug. 2007; S.L. Liu leg.; IZAS. Zhejiang • 1 đ̊; Qingyuan, Baishanzu; 1500 m a.s.l.; 9 Sep. 1993; H. Wu leg.; IZAS.

\section{Supplementary description}

AedeAgus (Fig. 5D-F). Strongly swollen dorsally in basal part, contracted in diameter basally; basal piece obviously shorter than dorsal plate of each paramere, with a large, bifurcate conjoint middle nodule at base of ventral side; ventral process of each paramere nearly parallel to one another, long and flattened, apex rounded; dorsal plate slightly shorter than ventral process, nearly parallel-sided, layered in middle, external layer roundly emarginate in middle, internal layer feebly emarginate, apex subrounded; apical parts of laterophyses compressed and next to each other in middle, apex rounded and obviously exceeding middle emargination between dorsal plates.

\section{Remarks}

In the original publication (Wittmer 1979), the aedeagus of this species was illustrated only in ventral view; here, it is also illustrated in dorsal and lateral views.

\section{Distribution}

China (Anhui, Fujian, Zhejiang).

\section{Key to the species of Stenothemus from Southeast China}

1. Elytra red, with moderately indicated longitudinal costae on disc

S. chinensis (Wittmer, 1982) comb. nov.

- Elytra black, yellow or light brown, with weakly or hardly indicated longitudinal costae on disc... 2

2. Pronotum subrounded, with lateral margins obviously sinuate, posterior angles distinctly protruding

- Pronotum subquadrate, with lateral margins nearly parallel or feebly sinuate, posterior angles hardly protruding

3. Elytra light brown, with irregular dark markings; aedeagus: dorsal plate of each paramere rounded at apex, layered but without protuberance at inner margin.

- Elytra yellow or black, without markings; aedeagus: dorsal plate of each paramere acute at apex, not layered, with a wide triangular protuberance at inner margin 
4. Elytra uniformly yellow.

S. biimpressiceps (Pic, 1930) comb. nov.

- Elytra black, with light yellow margins.....

S. fukienensis Wittmer, 1974

5. Antennae extending beyond elytral apices, antennomeres III-XI obviously widened and flattened in male.

S. longicornis Y. Yang \& H. Liu sp. nov.

- Antennae unlike above, at most reaching apical one-third length of elytra ...

6. Elytra yellow, black at apices; aedeagus: basal piece with a triangular protuberance but without middle nodule on ventral side, laterophyses hooked at apices ........S. nigriceps (Wittmer, 1955) comb. nov.

- Elytra unlike above, without apical macula; aedeagus: basal piece with a middle nodule on ventral side, laterophyses acute, never hooked.

7. Elytra black, at most light brown at margins; antennae with smooth longitudinal grooves on antennomeres III-XI in male S. limbatipennis (Pic, 1926) stat. rev. et comb. nov.

- Elytra yellow or light brown, at most with brown markings; antennae without smooth longitudinal grooves on antennomeres III-XI in male

8. Elytra uniformly yellow; aedeagus: dorsal plate of each paramere abruptly narrowed in middle...... S. flavus Y. Yang \& X. Yang sp. nov.

- Elytra light brown, with brown markings; aedeagus: dorsal plate of each paramere nearly parallelsided...... S. pallicolor (Wittmer, 1951) comb. nov.

\section{Discussion}

The diagnosis of Stenothemus was summarized by Okushima \& Satô (1999), but no characters of female genitalia were included. Here, the internal organ of the female reproductive system is described for the first time. All studied species share the following common characters: slender tube-shaped and spiral diverticulum and spermatheca, the situation of median oviduct, diverticulum and spermathecal duct in the vagina. They differ from one another in the relative length of diverticulum, spermathecal duct, spermatheca and accessory gland. It is shown once more that the female genitalia are a source of useful characters for cantharid taxonomy.

The female abdominal sternite VIII of Stenothemus, according to Okushima \& Satô (1999) lacks a membranous plate at the center of the terminal margin. However, this structure is present in the type species, S. harmandi (Bourgeois) (Yang et al. 2014), and the species studied here, except for S. nigriceps in which the membranous plate is absent.

Examination of the morphological characters of the types and a large series of additional material, especially characters of the male genitalia (Okushima \& Satô, 1999), including fused parameres being deeply cleft on the ventral side, and a pair of laterophyses present between the median lobe and dorsal plates that are moderately developed but much shorter than lateral lobes, with the basal part surmounting laterophyses more or less globularly swollen, indicated that the following species should be transferred to this genus: Stenothemus biimpressiceps (Pic, 1930) comb. nov. (from Cantharis Linnaeus, 1758), and Stenothemus chinensis (Wittmer, 1982) comb. nov., Stenothemus limbatipennis (Pic, 1926) stat. rev. et comb. nov., Stenothemus nigriceps (Wittmer, 1955) comb. nov. and Stenothemus pallicolor (Wittmer, 1951) comb. nov. (all from Lycocerus Gorham, 1889).

Based on the examination and comparison of the types as well as referring to some more additional material, Leiothorax atrosanguineus Švihla, 2005 is proposed as a junior synonym of Stenothemus chinensis. With the change in genus, S. limbatipennis is reinstated as a valid name, and Lycocerus limatus Kazantsev, 2007 becomes a junior synonym (ICZN Article 59.4). 


\section{Acknowledgements}

We are grateful to the late Dr Michel Brancucci (NHMB) for his great help to the senior author in studying the taxonomy of Cantharidae. Also, we are indebted to Prof. Hong Pang (SYSU), Dr David Kavanaugh (CAS) and Dr Antoine Mantilleri (MNHN) for allowing access to the cantharid types under their care. Special thanks are given to Mr John MacDermott (USA) for correcting our English in the manuscript, to Mr Aston Paul (Hong Kong, China) for presenting us with two valuable specimens and to Dr Wangang Liu (Institute of Earth Environment, Chinese Academy of Sciences, Xi'an, China) for his help in examining some types in ZFMK. The present study was supported by the National Natural Science Foundation of China (Nos 31772507, 41401064), the Natural Science Foundation of Hebei Province (C201720112,C2019201192), the Biodiversity Survey and Assessment Project of the Ministry of Ecology and Environment, China (2019HJ2096001006) and Post-graduate's Innovation Fund Project of Hebei University (HBU2021ss059) as well as the Science and Technology Project of Hebei Education Department (BJ2017030).

\section{References}

Brancucci M. 1980. Morphologie comparée, évolution et systématique des Cantharidae (Insecta: Coleoptera). Entomologica Basiliensia 5: 215-388.

Bourgeois J. 1907. Sur quelques Malacodermes de l'Inde. Annales de la Société entomologique de Belgique 51: 291-293.

Hsiao Y. 2015. Description of two new species of the genus Stenothemus from Taiwan (Coleoptera: Cantharidae). Zootaxa 3937 (2): 386-392. https://doi.org/10.11646/zootaxa.3937.2.9

Hsiao Y., Okushima Y. \& Yang P. 2016. Review of the genus Stenothemus Bourgeois from Taiwan, with description of three new species (Coleoptera: Cantharidae) and additional distributional records of previously known species. Zootaxa 4117 (1): 101-114. https://doi.org/10.11646/zootaxa.4117.1.5

Kazantsev S.V. 2007. Cantharidae. In: Löbl I \& Smetana A. (eds) Catalogue of Palaearctic Coleoptera 4: 47-54. Apollo Books, Stenstrup.

Kazantsev S.V. \& Brancucci, M. 2007. Cantharidae. In: Löbl I \& Smetana A. (eds) Catalogue of Palaearctic Coleoptera 4: 234-298. Apollo Books, Stenstrup.

Okushima Y. 2005. A taxonomic study on the genus Lycocerus (Coleoptera, Cantharidae) from Japan, with zoogeographical considerations. Japanese Journal of Systematic Entomology, Monographic Series 2: $1-383$.

Okushima Y. \& Satô M. 1997. Two new species of the genus Stenothemus (Coleoptera, Cantharidae) from Taiwan. Elytra 25 (1): 85-91.

Okushima Y. \& Satô M. 1999. Cantharid beetles of the genus Habronychus (Coleoptera, Cantharidae) from Taiwan, with description of a new subgenus. Elytra 27 (2): 387-403.

Pic M. 1906. Noms nouveaux et diagnoses de "Cantharini" (Telephorides) européens et exotiques. L'Échange 22: 81-85.

Pic M. 1930. Malacodermes exotiques (Suite). L'Échange 66 [hors-texte] (439): 77-80.

Švihla V. 2004. New taxa of the subfamily Cantharinae (Coleoptera, Cantharidae) from southeastern Asia with notes on other species. Entomologica Basiliensia 26: 155-238.

Švihla V. 2005. New taxa of the subfamily Cantharinae (Coleoptera: Cantharidae) from south-eastern Asia with notes on other species II. Acta Entomologica Musei Nationalis Pragae 45: 71-110. 
Švihla V. 2011. New taxa of the subfamily Cantharinae (Coleoptera: Cantharidae) from south-eastern Asia, with notes on other species III. Zootaxa 2895: 1-34.

Wittmer W. 1951. Neue Cantharidae aus Herrn Joh. Klapperichs' Südchina Ausbeute (14. Beitrag zur Kenntnis der palaearktischen Malacodermata Col.). Entomologische Blätter für Biologie und Systematik der Käfer 47: 96-103.

Wittmer W. 1955. 21. Beitrag zur Kenntnis der Palaearktischen Malacodermata (Coleoptera). Mushi 29 (6): 39-45.

Wittmer W. 1969. Synonymische und systematische Notizen über Coleopteren. Mitteilungen der Schweizerischen Entomologischen Gesellschaft 42 (1-2): 126-134.

https://doi.org/10.5169/SEALS-401586

Wittmer W. 1972. Beitrag zur Kenntnis der palaearktischen Cantharidae und Malachiidae (Col.). Entomologische Arbeiten aus dem Museum G. Frey 23: 122-141.

Wittmer W. 1974. Zur Kenntnis der Gattung Stenothemus Bourg. (Col. Cantharidae). Mitteilungen der Schweizerischen Entomologischen Gesellschaft 47 (1-2): 49-62.

Wittmer W. 1979. 64. Beitrag zur Kenntnis der palaearktischen Cantharidae, Phengodidae und Malachiidae (Col.). Entomologica Basiliensia 4: 327-346.

Wittmer W. 1982. 71. Beitrag zur Kenntnis der palaearktischen Cantharidae. Entomologica Basiliensia 7: 340-347.

Yang Y.X., Su J.Y. \& Yang X.K. 2014. Review of the Stenothemus harmandi species-group (Coleoptera, Cantharidae), with description of six new species from China. Zootaxa 3847 (2): 203-220.

https://doi.org/10.11646/zootaxa.3847.2.2

Manuscript received: 28 September 2020

Manuscript accepted: 25 January 2021

Published on: 12 April 2021

Topic editor: Nesrine Akkari

Section editor : Max Barclay

Desk editor: Kristiaan Hoedemakers

Printed versions of all papers are also deposited in the libraries of the institutes that are members of the EJT consortium: Muséum national d'histoire naturelle, Paris, France; Meise Botanic Garden, Belgium; Royal Museum for Central Africa, Tervuren, Belgium; Royal Belgian Institute of Natural Sciences, Brussels, Belgium; Natural History Museum of Denmark, Copenhagen, Denmark; Naturalis Biodiversity Center, Leiden, the Netherlands; Museo Nacional de Ciencias Naturales-CSIC, Madrid, Spain; Real Jardín Botánico de Madrid CSIC, Spain; Zoological Research Museum Alexander Koenig, Bonn, Germany; National Museum, Prague, Czech Republic. 